RESEARCH ARTICLE

\title{
Harmonic-enhanced high-gain harmonic generation for a high repetition rate free-electron laser
}

\author{
Sheng Zhao® ${ }^{1}$, Weilun Qin ${ }^{2}$, and Senlin Huang ${ }^{1}$ \\ ${ }^{1}$ State Key Laboratory of Nuclear Physics and Technology and Institute of Heavy Ion Physics, School of Physics, Peking University, \\ Beijing 100871, China \\ ${ }^{2}$ Deutsches Elektronen-Synchrotron (DESY), 22603 Hamburg, Germany \\ (Received 30 June 2021; revised 26 November 2021; accepted 6 December 2021)
}

\begin{abstract}
High-gain harmonic generation (HGHG) is effective to produce fully coherent free-electron laser (FEL) pulses for various scientific applications. Due to the limitation of seed lasers, HGHG typically operates at a low repetition rate. In this paper, a harmonic-enhanced HGHG scheme is proposed to relax the peak power requirement for the seed laser, which can therefore operate at megahertz and a higher repetition rate. Moreover, the setup of the scheme is compact and can be adopted in an existing single-stage HGHG facility to extend the shortest achievable wavelength. Simulations show that FEL emission at $13.5 \mathrm{~nm}$ (20th harmonic) can be obtained with a $270 \mathrm{~nm}, 1 \mathrm{MW}$ (peak power) seed laser.
\end{abstract}

Keywords: coherent radiation; extreme ultraviolet; free-electron laser; high-gain harmonic generation; high repetition rate

\section{Introduction}

High-brightness, highly stable and fully coherent pulses from high repetition rate free-electron lasers (FELs) in the extreme ultraviolet (EUV) and X-ray spectral regimes are capable of driving scientific applications ${ }^{[1,2]}$ such as timeresolved coherent spectroscopy ${ }^{[3]}$, photon scattering ${ }^{[4]}$ and coherent control ${ }^{[5,6]}$. Currently available megahertz repetition rate short-wavelength FEL user facilities, the European $\mathrm{XFEL}^{[7]}$ and $\mathrm{FLASH}^{[8]}$, both operate in burst mode. Several continuous wave $(\mathrm{CW})$ facilities are under construction, including the LCLS-II ${ }^{[9]}$ and SHINE ${ }^{[10]}$. One of the main operation modes for these FEL facilities is self-amplified spontaneous emission $(\mathrm{SASE})^{[11,12]}$. Originating from electron shot noise, SASE FELs have poor temporal coherence and large shot-to-shot fluctuations ${ }^{[13]}$.

For the current FEL facilities worldwide ${ }^{[12,14]}$, one effective method to improve the temporal coherence is adopting seeded schemes, which can be classified as self-seeding and external seeding. Compared with self-seeding schemes, external seeding schemes, including high-gain harmonic generation $(\mathrm{HGHG})^{[15-17]}$ and echo-enabled harmonic

Correspondence to: S. Huang, State Key Laboratory of Nuclear Physics and Technology and Institute of Heavy Ion Physics, School of Physics, Peking University, Beijing 100871, China. Email: huangsi@pku.edu.cn generation $(\mathrm{EEHG})^{[18-21]}$, can generate FEL pulses with lower energy fluctuation. They also provide extra capabilities to be highly synchronized with the external laser and to control the coherence in multi-color, multi-pulse implementations ${ }^{[6,22]}$. These fulfill the requirements of the aforementioned scientific applications.

In HGHG FELs ${ }^{[15]}$, a sinusoidal energy modulation is first introduced to the electrons via the interaction between the electron beam and an external seed laser within a modulation undulator (called a modulator). After a magnetic chicane, the energy modulation is converted to density modulation, creating micro-bunching at the harmonic of the seed laser wavelength. The bunched beam is then delivered to aradiation undulator (called a radiator) resonant at the harmonic wavelength. To obtain sufficient bunching at a higher harmonic, the amplitude of the energy modulation imprinted by the seed laser needs to be large enough. However, for efficient FEL amplification in the radiator, the induced electron beam energy spread should be kept at a low level. This, in turn, places a restriction on the highest achievable harmonic for an HGHG FEL. For example, for the FERMI FEL-1, the highest harmonic for FEL emission was the 13th harmonic, although coherent emission could be observed at a higher (15th) harmonic ${ }^{[17]}$. Recently, the operation range of the FERMI FEL-1 was extended to the 25th harmonic 
by employing an electron beam with a low slice energy spread $^{[23]}$.

For high repetition rate HGHG FELs, the external laser becomes a determinant at the megahertz repetition rate. The state-of-the-art laser system capable of delivering the hundred-megawatt pulses required by an HGHG scheme is limited to the kilohertz repetition rate. Several methods have been proposed to relax the peak power or repetition rate requirements for the seed laser ${ }^{[24]}$, including using an optical cavity for seed laser recirculation and/or amplification $^{[25,26]}$ and employing a long modulator for both seed laser amplification and electron beam modulation ${ }^{[27-29]}$. To enhance the energy modulation efficiency, a self-modulation HGHG scheme was also proposed ${ }^{[27,30]}$, which was demonstrated at the Shanghai Soft X-ray Free-electron Laser facility (SXFEL) recently ${ }^{[31,32]}$. With a single-stage HGHG setup, the seventh harmonic emission has been obtained, while with a two-stage cascaded HGHG setup and the 'fresh bunch' technique, the 30th harmonic emission has been obtained. It is worth noting that the self-modulator was also proposed to resonate at the second harmonic of the seed laser wavelength in Refs. [27,31], in which case the 10th harmonic emission could be obtained according to simulation.

In this paper, we study a compact harmonic-enhanced HGHG scheme to further extend the highest harmonic for FEL emission in a high repetition rate, single-stage HGHG scheme. Compared to a regular HGHG scheme, an additional modulation undulator resonant at a high-harmonic wavelength is introduced, which essentially causes efficient electron density modulation up to the 20th harmonic wavelength, but with a relaxed peak power requirement for the seed laser and reduced electron energy spread growth. Moreover, since the electrons are bunched at a high harmonic of the seed laser wavelength, the density modulation can be achieved via a short dispersion section. This ensures the compactness of the proposed scheme.

The remainder of this paper is organized as follows. In Section 2, we give a detailed description of the scheme. In Section 3, the performance of the scheme is illustrated with simulations. Section 4 gives a summary and some discussions.

\section{The scheme}

A layout of the proposed scheme is sketched in Figure 1, which mainly comprises a short modulation undulator $U_{0}$ resonant at the seed laser wavelength $\lambda$ (referred to as the 'fundamental modulator' hereafter), a four-dipole magnet chicane $C_{1}$, a second modulation undulator $U_{1}$ resonant at the $n$th harmonic of $\lambda$ (referred to as the 'harmonicenhanced modulator' hereafter), a short dispersion section $C_{2}$ and a radiation undulator resonant at the $(m \times n)$ th harmonic of $\lambda$ (referred to as the 'radiator' hereafter). The electron beam first interacts with the low-power seed laser in the fundamental modulator. This interaction leads to a weak energy modulation to the electrons at the scale of $\lambda$. Afterwards, the electron beam traverses the four-dipole magnet chicane $C_{1}$, through which the fundamental energy modulation is converted to longitudinal density modulation with low-order harmonic components. The electron beam is then injected into the harmonic-enhanced modulator $U_{1}$, where the electrons emit coherent radiation at $\lambda / n$. This in turn modulates the electrons at the scale of $\lambda / n$. The harmonic energy modulation can be transformed into density modulation via $C_{2}$ and now the electron current distribution has a significant harmonic component at $\lambda / m n$. The modulated electron beam is finally sent to the radiator $U_{2}$ and emits FEL radiation at $\lambda / m n$.

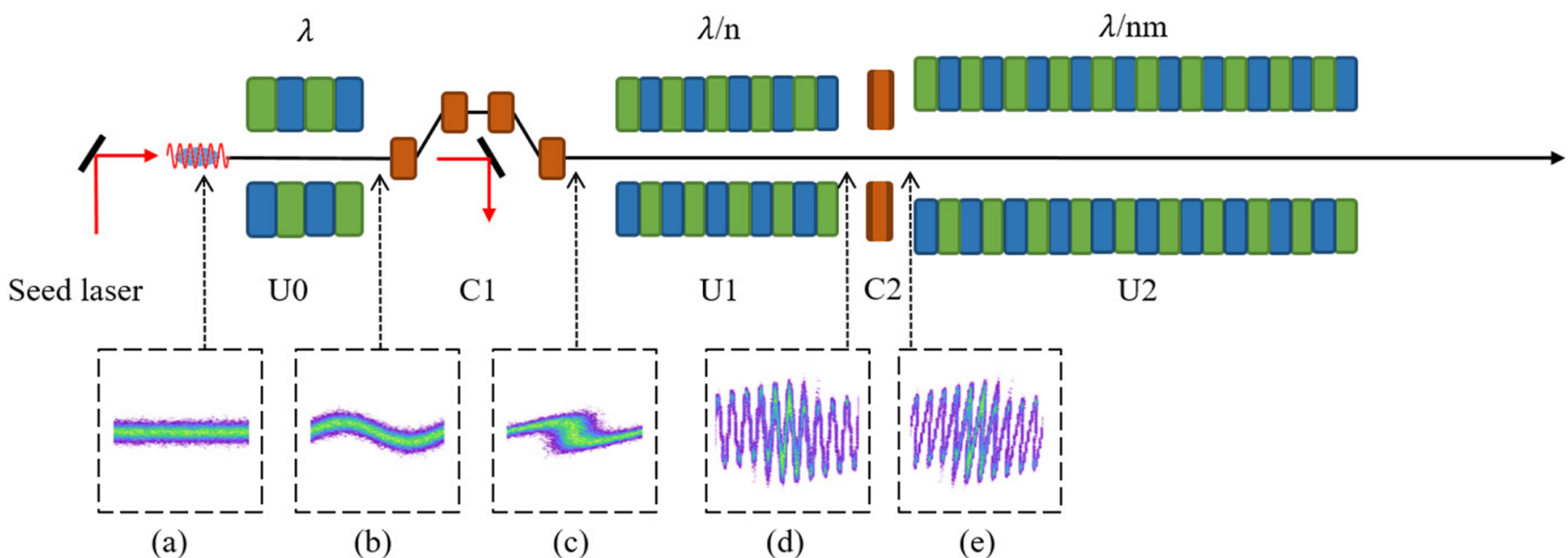

Figure 1. Schematic layout of the harmonic-enhanced HGHG FEL. Here, $U_{0}$ is a short modulation undulator, where an external seed laser with a wavelength of $\lambda$ and a peak power at the megawatt level is used to modulate the electron beam; $U_{1}$ is a harmonic-enhanced modulation undulator resonant at $\lambda / n$ and $U_{2}$ is a radiation undulator resonant at $\lambda / \mathrm{nm} ; C_{1}$ and $C_{2}$ are two dispersion chicanes. (a)-(e) Sketches of electron beam distribution in the longitudinal phase space. 
A similar layout can be found in the scheme of self-modulation $\mathrm{HGHG}^{[31]}$, where the first stage of the HGHG radiator is resonant at the fundamental or the second harmonic wavelength. Our work can be distinguished by the high-order harmonic modulator and the significant bunching factor up to the 20th harmonic of the seed laser wavelength. Another scheme with a similar layout is the double-stage harmonic cascade HGHG demonstrated at the FERMI FEL to extend the wavelength coverage of the HGHG scheme ${ }^{[33]}$. However, the first-stage radiator resonates at a much higher harmonic (13th) wavelength, which then requires a higher seed laser power for the high repetition rate operation.

For sufficient FEL emission at harmonic wavelength, it is essential to induce effective electron bunching at the target wavelength while keeping the energy spread growth as low as possible. In this scheme, the electron beam undergoes two-stage modulation, one in the fundamental modulator $U_{0}$ and the other in the harmonic-enhanced modulator $U_{1}$. Both modulation processes will induce extra energy spread. However, as the modulation wavelength becomes shorter, the required energy modulation amplitude for the same bunching factor (after dispersion) becomes lower. Therefore, the induced energy spread by the two-stage modulation is at a low level.

The setup of the scheme can be very compact. Since the optimal dispersion of $C_{2}$ is proportional to the modulation wavelength $\lambda / n$ and inversely proportional to the modulation amplitude ${ }^{[34]}$, the required dispersion can be provided by a small magnetic chicane, which can be inserted between the undulator segments of regular FELs. Similar small chicanes have been used in Refs. [35,36]. Moreover, since undulators have weak dispersion, the setup can be simplified by removing $C_{2}$. This compactness allows adopting the proposed scheme in an existing single-stage HGHG facility to extend the shortest achievable wavelength.

\section{FEL simulations of harmonic-enhanced HGHG}

To demonstrate the feasibility of the proposed scheme, time-dependent FEL simulations using GENESIS code ${ }^{[37]}$ have been carried out, with the representative parameters summarized in Table 1 . The electron beam was assumed to have a central energy of $1 \mathrm{GeV}$, a slice energy spread $\left(\sigma_{\mathrm{E}}\right)$ of $0.1 \mathrm{MeV}$, a peak current of $800 \mathrm{~A}$, a bunch charge of $100 \mathrm{pC}$ and a normalized transverse emittance of $1 \mathrm{~mm} \cdot \mathrm{mrad}$. The fundamental modulator $U_{0}$ was assumed to have a period of $8 \mathrm{~cm}$ and a total length of $3.2 \mathrm{~m}$. The harmonic-enhanced modulator $U_{1}$ and the radiator $U_{2}$ both have a period of $4 \mathrm{~cm}$ and each undulator segment has a length of $3 \mathrm{~m}$. The seed laser was assumed to have a central wavelength $\left(\lambda_{\mathrm{s}}\right)$ of $270 \mathrm{~nm}$ and a peak power of $1 \mathrm{MW}$. Such a laser at the $\mathrm{MHz}$ repetition rate is commercially available ${ }^{[38]}$.

For the proposed scheme, a proper choice of the harmonic number $n$ for the harmonic-enhanced modulator is of
Table 1. Main parameters used in the simulations.

\begin{tabular}{lcc}
\hline Parameter & Value & Unit \\
\hline Electron beam & 1 & $\mathrm{GeV}$ \\
Energy & 0.1 & $\mathrm{MeV}$ \\
Energy spread & 800 & $\mathrm{~A}$ \\
Current & 1 & $\mathrm{~mm} \cdot \mathrm{mrad}$ \\
Emittance & 100 & $\mathrm{pC}$ \\
Bunch charge & & $\mathrm{nm}$ \\
Seed laser & 270 & $\mathrm{MW}$ \\
Wavelength & 1 & $\mathrm{fs}$ \\
Peak power & 150 & $\mathrm{~cm}$ \\
Pulse length & & $\mathrm{m}$ \\
Undulator & 8 & $\mathrm{~cm}$ \\
$U_{0}$ period & 3.2 & $\mathrm{~m}$ \\
$U_{0}$ length & 4 & \\
$U_{1} / U_{2}$ period & 3 & \\
$U_{1} / U_{2}$ segment length & &
\end{tabular}

importance. A large $n$ demands a seed laser with high peak power and also leads to significant energy spread growth in the fundamental modulator. On the other hand, when $n$ is small, a large energy spread growth will be induced in the harmonic-enhanced modulator so as to imprint effective bunching at the target wavelength. As a compromise between the seed laser power and the induced energy spread growth, the harmonic number $n$ was chosen to be 5 in our case.

Similarly, the length of the harmonic-enhanced modulator should also be chosen properly. On one hand, $U_{1}$ should be long enough to assure sufficient interaction between the electrons and the coherent radiation so as to imprint enough electron energy modulation at the $n$th harmonic of the seed laser wavelength. However, on the other hand, a strong modulation would inevitably lead to the degradation of electron beam quality, which will degrade the FEL performance in the radiator. As a compromise, $U_{1}$ was chosen to have two undulator segments with a total length of $6 \mathrm{~m}$.

In this case, the interaction between the seed laser and the electron beam in $U_{0}$ introduces an energy modulation amplitude of $2.1 \sigma_{\mathrm{E}}$. The dispersion strength $\left(R_{56}\right)$ of $C_{1}$ was accordingly optimized for the maximized bunching factor at the fifth harmonic wavelength. The longitudinal phase space of the electron beam after $C_{1}$ is shown in Figure 2(a) and the corresponding bunching factor as a function of the harmonic number is plotted in Figure 2(b). As shown in the figure, the bunching factor is 0.009 at the fifth harmonic, while the bunching factor at the 20th harmonic is at the shot noise level.

The longitudinal phase space after $U_{1}$ is shown in Figure 2(c), where an energy modulation amplitude of $7.4 \sigma_{\mathrm{E}}$ induced by the harmonic-enhanced process can be observed. Due to the weak dispersion of $U_{1}$, the energy modulation is partially converted to density modulation. As shown in Figure 2(d), the increase of the bunching factors can be observed at the integer multiple of the fifth harmonic. The bunching factor at the fifth and 20th harmonics increases to 0.17 and 0.003 , respectively. 

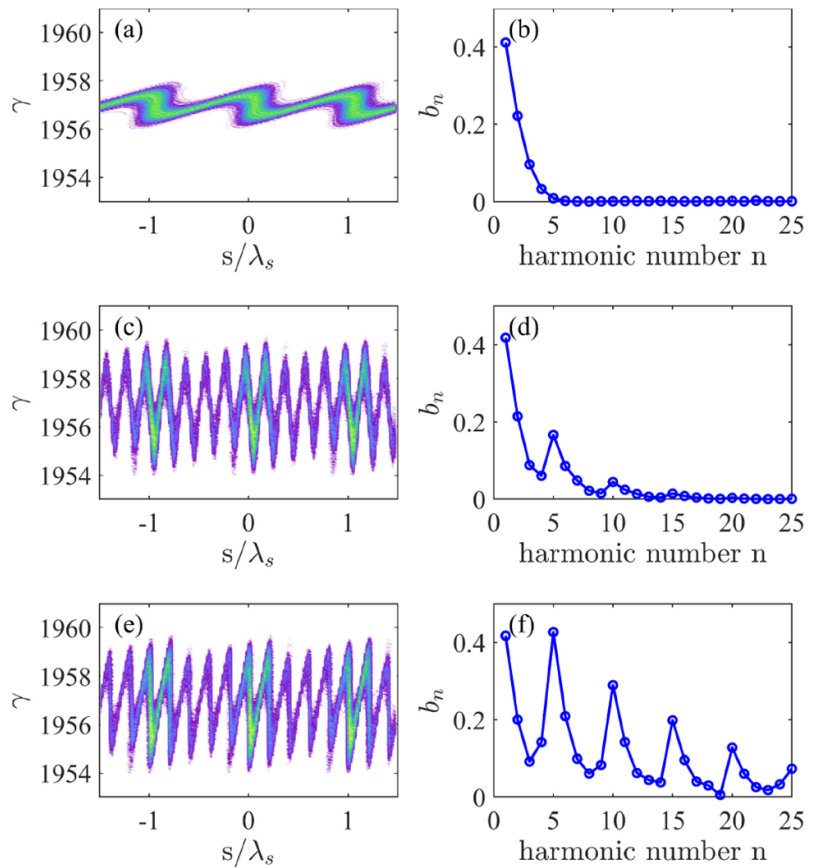

Figure 2. Electron distribution in the longitudinal phase space and the corresponding bunching factor at different harmonics of the seed laser wavelength after (a), (b) $C_{1}$, (c), (d) $U_{1}$ and (e), (f) $C_{2}$.

To fully convert the electron energy modulation after $U_{1}$ to density modulation, the $R_{56}$ of $C_{2}$ was set to be $8.6 \mu \mathrm{m}$. Figures 2(e) and 2(f) show the longitudinal phase space and the corresponding bunching factor after $C_{2}$. A bunching factor of 0.13 has been achieved at the 20th harmonic. It is worth noting that the harmonic-enhanced modulator introduces electron energy modulation at the fifth harmonic wavelength of the seed laser, and after $C_{2}$, the electrons are micro-bunched at $\lambda_{\mathrm{s}} / 5$. Therefore, the bunching factor reaches maxima at the harmonics of $\lambda_{\mathrm{s}} / 5$, for example, the 10th, 15th and 20th harmonics of the seed laser wavelength.

Fifty GENESIS runs were then performed with different random shot noise initializations and with the radiator resonant at the wavelength of $13.5 \mathrm{~nm}\left(\lambda_{\mathrm{s}} / 20\right)$. The evolution of the FEL pulse energy along $U_{2}$ (averaged over the 50 runs) is presented in Figure 3(a) together with the evolution of harmonic radiation energy at $13.5 \mathrm{~nm}$ in $U_{1}$. As shown in the figure, at the exit of $U_{1}$ the radiation energy is about $16 \mathrm{~nJ}$, and in the radiator $U_{2}$, driven by the strong microbunching, the pulse energy grows exponentially and reaches saturation at $13.6 \mathrm{~m}$ with a pulse energy of $193 \mu \mathrm{J}$. The total length of $U_{1}, U_{2}$ and the break sections is about $21 \mathrm{~m}$, implying a rather compact configuration. The spectra for the 50 GENESIS runs and the average spectrum are plotted in Figure 3(b), indicating high stability and excellent temporal coherence. The average relative bandwidth (full width at half maximum, FWHM) is $3.7 \times 10^{-4}$, which is comparable to regular HGHG FELs ${ }^{[17,23]}$.
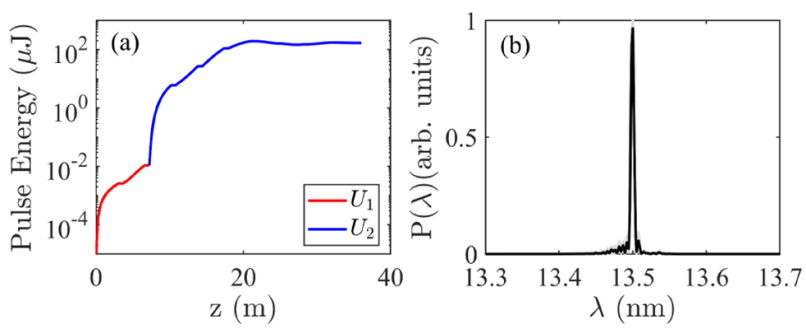

Figure 3. (a) The evolution of radiation pulse energy along $U_{1}$ and $U_{2}$ averaged over 50 GENESIS runs and (b) the FEL spectra for the 50 runs for the case with $C_{2}$. The averaged spectrum is plotted as a dark line in (b).

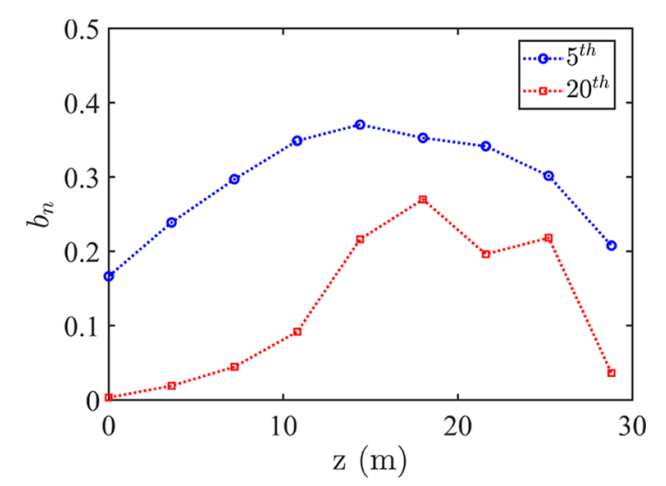

Figure 4. Bunching factor evolution at the fifth and 20th harmonics of seed laser wavelength along the radiation undulator $U_{2}$ for the case without $C_{2}$.

To facilitate the estimation of repetition rate improvement of the proposed scheme compared to a regular HGHG scheme, we simulated a regular HGHG scheme using the same parameters for the modulator $U_{0}$. In order to reach the same bunching factor of 0.13 at the 20th harmonic, it is found that a more than $400 \mathrm{MW}$ seed laser is needed. However, the induced energy spread is up to $40 \sigma_{\mathrm{E}}$, which prevents the radiation from entering the exponential gain regime. Therefore, assuming given average power for the seed laser, we estimate that the improvement of the repetition rate of the proposed scheme compared with a regular HGHG scheme is more than two orders of magnitude.

We also investigated the case without $C_{2}$ while all other parameters were kept unchanged. According to Ref. [39], the dispersion strength is about $2 \mu \mathrm{m}$ for each undulator segment of $U_{2}$. This undulator dispersion, together with the enhanced electron-radiation interaction, could lead to the increase of the bunching factor. The simulation result presented in Figure 4 shows the sustained growth of the bunching factors at the fifth and 20th harmonics in $U_{2}$ before the FEL reaches saturation. After saturation, the bunching factors begin to drop, mainly due to the distortion of electron distribution in the longitudinal phase space. It is worth noting that, after the first undulator segment of $U_{2}$, the bunching factor at the 20th harmonic grows to 0.02 , which indicates that the micro-bunching induced coherent emission dominates the FEL process in $U_{2}$. 

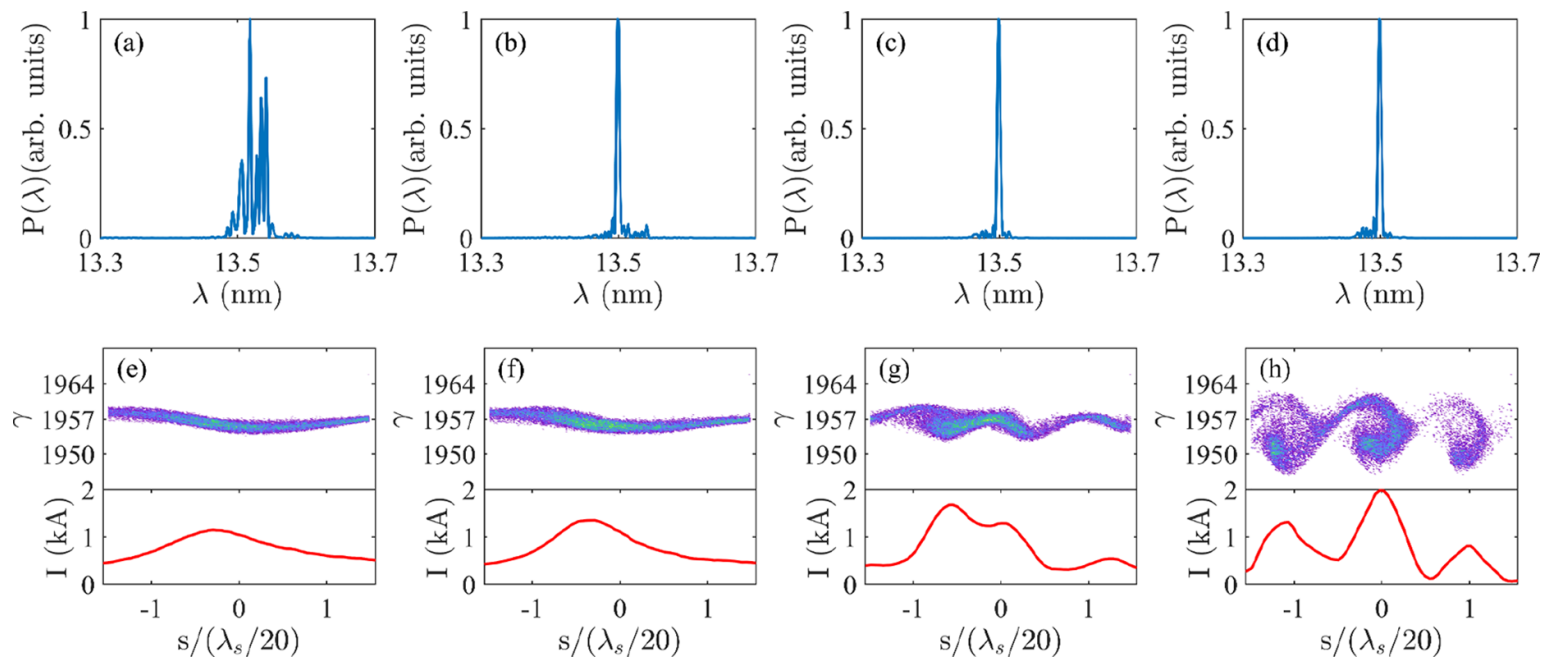

Figure 5. Evolution of the radiation spectra (upper) and the longitudinal phase space of the electron beam (lower) along $U_{2}$ : (a) and (e) at the entrance of $U_{2}$, (b) and (f) after the first undulator segment, (c) and (g) after the third segment and (d) and (h) after the fifth segment. The longitudinal coordinates are scaled to $\lambda_{\mathrm{s}} / 20$. The electron current profiles are plotted as red curves in (e)-(h).

The evolutions of the radiation spectrum, electron phase space and beam current profile along $U_{2}$ are illustrated in Figure 5. The spectra indicate that the coherent emission dominates after one undulator segment. This is consistent with the previous observation. Comparing the phase space distribution in Figures 5(e) and 5(f), we can also observe the conversion from electron energy modulation to density modulation due to the dispersion of the undulator. Driven by the growing coherent emission from the bunched beam, micro-bunching at $\lambda_{\mathrm{s}} / 20$ becomes stronger, as shown in Figures $5(\mathrm{~g})$ and $5(\mathrm{~h})$, which accounts for the fast growth of the bunching factor at the 20th harmonic, as shown in Figure 4.

Fifty GENESIS runs have been performed as well, and the results are plotted in Figure 6. As shown in Figure 6(a), the FEL saturates at $21.6 \mathrm{~m}$ of $U_{2}$, which is a little longer than the previous case with the dispersion chicane $C_{2}$. The saturation pulse energy (averaged over the 50 runs), $176 \mu \mathrm{J}$, is also a little bit lower. The spectra for the 50 runs [see Figure 6(b)] are less stable but still show good temporal coherence. The average relative bandwidth (FWHM) is $3.6 \times 10^{-4}$, similar to the previous case.

We finally investigated the robustness of the scheme against machine errors. For the simulation, we used the configuration without $C_{2}$. A series of 200 separate GENESIS runs were performed with a random variation of the seed laser power within $1 \%, R_{56}$ within $1 \%$, electron peak current within $1 \%$, electron central energy within $0.05 \%$, electron energy spread within $1 \%$ and undulator strength within $0.01 \%$ (for each segment), which are feasible for most existing facilities ${ }^{[40,41]}$. The results are shown in Figure 7. The average pulse energy over 200 runs is $166 \mu \mathrm{J}$ and the relative jitter of the pulse energy is $7.8 \%$ (root mean square, RMS), which illustrate the stability of the proposed scheme.
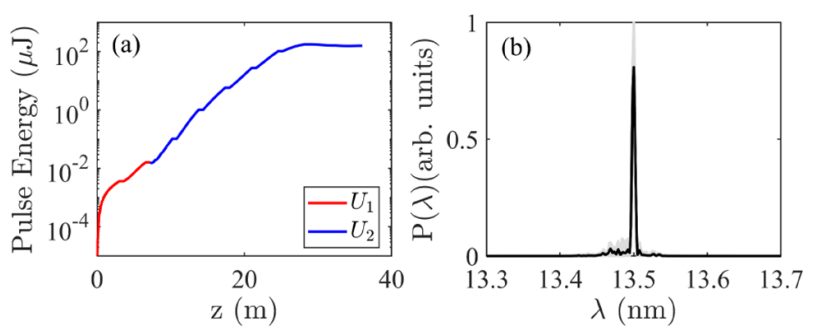

Figure 6. (a) The evolution of radiation pulse energy along $U_{1}$ and $U_{2}$ averaged over 50 GENESIS runs and (b) the FEL spectra for the 50 runs for the case without $C_{2}$. The averaged spectrum is plotted as a dark line in (b).

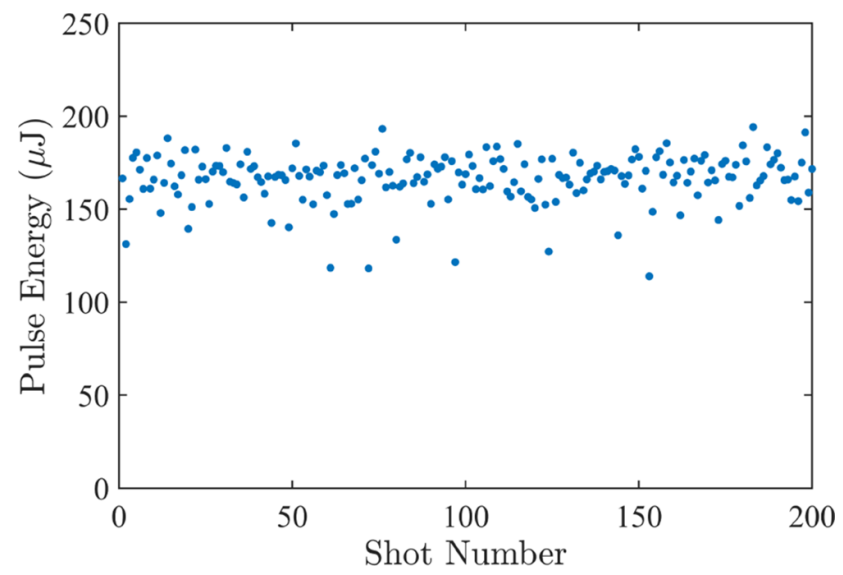

Figure 7. FEL pulse energy for 200 GENESIS runs with random machine errors.

The radiation bandwidth of the proposed scheme is also sensitive to the nonlinear energy chirp, as is a normal HGHG scheme. Some methods have been developed to control the nonlinear energy chirp at a low level ${ }^{[42]}$. With an assumed $0.3 \mathrm{MeV} / \mathrm{ps}^{2}$ quadratic energy chirp, the bandwidth will 
be increased to $0.13 \%{ }^{[43]}$, which is about 10 times the transform-limited for the same pulse length.

\section{Summary and discussion}

In summary, we have studied a robust harmonic-enhanced HGHG scheme to generate high repetition rate coherent FEL using a low peak power seed laser. The performance for two possible configurations has been investigated using GENESIS simulations, one with a short dispersion chicane after the harmonic-enhanced modulator and the other without the chicane. Both configurations can generate coherent FEL output at $13.5 \mathrm{~nm}$ with a $270 \mathrm{~nm}, 1 \mathrm{MW}$ (peak power) seed laser. For the case with the dispersion chicane, a bunching factor of 0.13 can be achieved at the 20th harmonic of the seed laser wavelength before the electron beam enters the radiator, and the FEL saturates at about $13.6 \mathrm{~m}$ in the radiator with a pulse energy of $193 \mu \mathrm{J}$. The case without the dispersion chicane has a longer saturation length of $21.6 \mathrm{~m}$ and a lower pulse energy of $176 \mu \mathrm{J}$. However, it is only necessary to tune a few radiation undulator segments in a regular HGHG scheme for it to be resonant at a higher harmonic (e.g., fifth harmonic in our simulation) of the fundamental wavelength. The length of these undulator segments (i.e., the harmonic-enhanced modulator) can be varied easily, providing the flexibility of optimizing the harmonicenhanced modulator for different harmonics. In particular, with a longer modulator, even higher harmonics for soft $\mathrm{X}$-ray emission might be reached. Besides, a few methods can be used to further improve the FEL performance. For example, the first undulator segment of $U_{2}$ can be reversely tapered, so that it mainly acts as a dispersive section ${ }^{[44]}$, which might shorten the FEL saturation length significantly. It is also possible to further increase the FEL pulse energy by tapering the radiation undulator ${ }^{[45,46]}$. Also note that the harmonic-enhanced modulator proposed in this paper can be used to extend the achievable harmonic number of other seeded FELs, such as EEHG and self-seeded $\mathrm{HGHG}^{[28]}$.

\section{Acknowledgement}

This work was supported by the National Natural Science Foundation of China (No. 11975039).

\section{References}

1. E. A. Seddon, J. A. Clarke, D. J. Dunning, C. Masciovecchio, C. J. Milne, F. Parmigiani, D. Rugg, J. C. H. Spence, N. R. Thompson, K. Ueda, S. M. Vinko, J. S. Wark, and W. Wurth, Rep. Prog. Phys. 80, 115901 (2017).

2. N. Huang, H. Deng, B. Liu, D. Wang, and Z. Zhao, Innovation 2, 2 (2021).

3. M. Dell'Angela, F. Hieke, F. Sorgenfrei, N. Gerken, M. Beye, N. Gerasimova, H. Redlin, and W. Wurth, Surf. Sci. 643, 197 (2016).
4. R. R. Fäustlin, T. Bornath, T. Döppner, S. Düsterer, E. Förster, C. Fortmann, S. H. Glenzer, S. Göde, G. Gregori, R. Irsig, T. Laarmann, H. J. Lee, B. Li, K. H. Meiwes-Broer, J. Mithen, B. Nagler, A. Przystawik, H. Redlin, R. Redmer, H. Reinholz, G. Röpke, F. Tavella, R. Thiele, J. Tiggesbäumker, S. Toleikis, I. Uschmann, S. M. Vinko, T. Whitcher, U. Zastrau, B. Ziaja, and T. Tschentscher, Phys. Rev. Lett. 104, 125002 (2010).

5. K. C. Prince, E. Allaria, C. Callegari, R. Cucini, G. De Ninno, S. Di Mitri, B. Diviacco, E. Ferrari, P. Finetti, D. Gauthier, L. Giannessi, N. Mahne, G. Penco, O. Plekan, L. Raimondi, P. Rebernik, E. Roussel, C. Svetina, M. Trovò, M. Zangrando, M. Negro, P. Carpeggiani, M. Reduzzi, G. Sansone, A. N. Grum-Grzhimailo, E. V. Gryzlova, S. I. Strakhova, K. Bartschat, N. Douguet, J. Venzke, D. Iablonskyi, Y. Kumagai, T. Takanashi, K. Ueda, A. Fischer, M. Coreno, F. Stienkemeier, Y. Ovcharenko, T. Mazza, and M. Meyer, Nat. Photonics 10, 176 (2016).

6. P. K. Maroju, C. Grazioli, M. Di Fraia, M. Moioli, D. Ertel, H. Ahmadi, O. Plekan, P. Finetti, E. Allaria, L. Giannessi, G. De Ninno, C. Spezzani, G. Penco, S. Spampinati, A. Demidovich, M. B. Danailov, R. Borghes, G. Kourousias, C. E. Sanches Dos Reis, F. Billé, A. A. Lutman, R. J. Squibb, R. Feifel, P. Carpeggiani, M. Reduzzi, T. Mazza, M. Meyer, S. Bengtsson, N. Ibrakovic, E. R. Simpson, J. Mauritsson, T. Csizmadia, M. Dumergue, S. Kühn, H. Nandiga Gopalakrishna, D. You, K. Ueda, M. Labeye, J. E. Bækhøj, K. J. Schafer, E. V. Gryzlova, A. N. Grum-Grzhimailo, K. C. Prince, C. Callegari, and G. Sansone, Nature 578, 386 (2020).

7. D. Nölle, in Proceedings of the 39th International Free Electron Laser Conference (2019), p. 766.

8. B. Faatz, E. Plönjes, S. Ackermann, A. Agababyan, V. Asgekar, V. Ayvazyan, S. Baark, N. Baboi, V. Balandin, N. Bargen, Y. Bican, O. Bilani, J. Bödewadt, M. Böhnert, R. Böspflug, S. Bonfigt, H. Bolz, F. Borges, O. Borkenhagen, M. Brachmanski, M. Braune, A. Brinkmann, O. Brovko, T. Bruns, P. Castro, J. Chen, M. K. Czwalinna, H. Damker, W. Decking, M. Degenhardt, A. Delfs, T. Delfs, H. Deng, M. Dressel, H. T. Duhme, S. Düsterer, H. Eckoldt, A. Eislage, M. Felber, J. Feldhaus, P. Gessler, M. Gibau, N. Golubeva, T. Golz, J. Gonschior, A. Grebentsov, M. Grecki, C. Grün, S. Grunewald, K. Hacker, L. Hänisch, A. Hage, T. Hans, E. Hass, A. Hauberg, O. Hensler, M. Hesse, K. Heuck, A. Hidvegi, M. Holz, K. Honkavaara, H. Höppner, A. Ignatenko, J. Jäger, U. Jastrow, R. Kammering, S. Karstensen, A. Kaukher, H. Kay, B. Keil, K. Klose, V. Kocharyan, M. Köpke, M. Körfer, W. Kook, B. Krause, O. Krebs, S. Kreis, F. Krivan, J. Kuhlmann, M. Kuhlmann, G. Kube, T. Laarmann, C. Lechner, S. Lederer, A. Leuschner, D. Liebertz, J. Liebing, A. Liedtke, L. Lilje, T. Limberg, D. Lipka, B. Liu, B. Lorbeer, K. Ludwig, H. Mahn, G. Marinkovic, C. Martens, F. Marutzky, M. Maslocv, D. Meissner, N. Mildner, V. Miltchev, S. Molnar, D. Mross, F. Müller, R. Neumann, P. Neumann, D. Nölle, F. Obier, M. Pelzer, H. B. Peters, K. Petersen, A. Petrosyan, G. Petrosyan, L. Petrosyan, V. Petrosyan, A. Petrov, S. Pfeiffer, A. Piotrowski, Z. Pisarov, T. Plath, P. Pototzki, M. J. Prandolini, J. Prenting, G. Priebe, B. Racky, T. Ramm, K. Rehlich, R. Riedel, M. Roggli, M. Röhling, J. Rönsch-Schulenburg, J. Rossbach, V. Rybnikov, J. Schäfer, J. Schaffran, H. Schlarb, G. Schlesselmann, M. Schlösser, P. Schmid, C. Schmidt, F. SchmidtFöhre, M. Schmitz, E. Schneidmiller, A. Schöps, M. Scholz, S. Schreiber, K. Schütt, U. Schütz, H. Schulte-Schrepping, M. Schulz, A. Shabunov, P. Smirnov, E. Sombrowski, A. Sorokin, B. Sparr, J. Spengler, M. Staack, M. Stadler, C. Stechmann, B. Steffen, N. Stojanovic, V. Sychev, E. Syresin, T. Tanikawa, F. Tavella, N. Tesch, K. Tiedtke, M. Tischer, R. Treusch, S. Tripathi, P. Vagin, P. Vetrov, S. Vilcins, M. Vogt, A. Z. Wagner, T. Wamsat, H. Weddig, G. Weichert, H. 
Weigelt, N. Wentowski, C. Wiebers, T. Wilksen, A. Willner, K. Wittenburg, T. Wohlenberg, J. Wortmann, W. Wurth, M. Yurkov, I. Zagorodnov, and J. Zemella, New J. Phys. 18, 062002 (2016).

9. J. N. Galayda, in Proceedings of the 9th International Particle Accelerator Conference (2018), p. 18.

10. Z. Zhu, Z. T. Zhao, D. Wang, Z. H. Yang, and L. Yin, in Proceedings of the 38th International Free Electron Laser Conference (2017), p. 182.

11. W. Ackermann, G. Asova, V. Ayvazyan, A. Azima, N. Baboi, J. Bähr, V. Balandin, B. Beutner, A. Brandt, A. Bolzmann, R. Brinkmann, O. I. Brovko, M. Castellano, P. Castro, L. Catani, E. Chiadroni, S. Choroba, A. Cianchi, J. T. Costello, D. Cubaynes, J. Dardis, W. Decking, H. Delsim-Hashemi, A. Delserieys, G. Di Pirro, M. Dohlus, S. Düsterer, A. Eckhardt, H. T. Edwards, B. Faatz, J. Feldhaus, K. Flöttmann, J. Frisch, L. Fröhlich, T. Garvey, U. Gensch, C. Gerth, M. Görler, N. Golubeva, H. J. Grabosch, M. Grecki, O. Grimm, K. Hacker, U. Hahn, J. H. Han, K. Honkavaara, T. Hott, M. Hüning, Y. Ivanisenko, E. Jaeschke, W. Jalmuzna, T. Jezynski, R. Kammering, V. Katalev, K. Kavanagh, E. T. Kennedy, S. Khodyachykh, K. Klose, V. Kocharyan, M. Körfer, M. Kollewe, W. Koprek, S. Korepanov, D. Kostin, M. Krassilnikov, G. Kube, M. Kuhlmann, C. L. S. Lewis, L. Lilje, T. Limberg, D. Lipka, F. Löhl, H. Luna, M. Luong, M. Martins, M. Meyer, P. Michelato, V. Miltchev, W. D. Möller, L. Monaco, W. F. O. Müller, O. Napieralski, O. Napoly, P. Nicolosi, D. Nölle, T. Nuñez, A. Oppelt, C. Pagani, R. Paparella, N. Pchalek, J. Pedregosa-Gutierrez, B. Petersen, B. Petrosyan, G. Petrosyan, L. Petrosyan, J. Pflüger, E. Plönjes, L. Poletto, K. Pozniak, E. Prat, D. Proch, P. Pucyk, P. Radcliffe, H. Redlin, K. Rehlich, M. Richter, M. Roehrs, J. Roensch, R. Romaniuk, M. Ross, J. Rossbach, V. Rybnikov, M. Sachwitz, E. L. Saldin, W. Sandner, H. Schlarb, B. Schmidt, M. Schmitz, P. Schmüser, J. R. Schneider, E. A. Schneidmiller, S. Schnepp, S. Schreiber, M. Seidel, D. Sertore, A. V. Shabunov, C. Simon, S. Simrock, E. Sombrowski, A. A. Sorokin, P. Spanknebel, R. Spesyvtsev, L. Staykov, B. Steffen, F. Stephan, F. Stulle, H. Thom, K. Tiedtke, M. Tischer, S. Toleikis, R. Treusch, D. Trines, I. Tsakov, E. Vogel, T. Weiland, H. Weise, M. Wellhöfer, M. Wendt, I. Will, A. Winter, K. Wittenburg, W. Wurth, P. Yeates, M. V. Yurkov, I. Zagorodnov, and K. Zapfe, Nat. Photonics 1, 336 (2007)

12. P. Emma, R. Akre, J. Arthur, R. Bionta, C. Bostedt, J. Bozek, A. Brachmann, P. Bucksbaum, R. Coffee, F. J. Decker, Y. Ding, D. Dowell, S. Edstrom, A. Fisher, J. Frisch, S. Gilevich, J. Hastings, G. Hays, P. Hering, Z. Huang, R. Iverson, H. Loos, M. Messerschmidt, A. Miahnahri, S. Moeller, H. D. Nuhn, G. Pile, D. Ratner, J. Rzepiela, D. Schultz, T. Smith, P. Stefan, H. Tompkins, J. Turner, J. Welch, W. White, J. Wu, G. Yocky, and J. Galayda, Nat. Photonics 4, 641 (2010).

13. D. Ratner, R. Abela, J. Amann, C. Behrens, D. Bohler, G. Bouchard, C. Bostedt, M. Boyes, K. Chow, D. Cocco, F. J. Decker, Y. Ding, C. Eckman, P. Emma, D. Fairley, Y. Feng, C. Field, U. Flechsig, G. Gassner, J. Hastings, P. Heimann, Z. Huang, N. Kelez, J. Krzywinski, H. Loos, A. Lutman, A. Marinelli, G. Marcus, T. Maxwell, P. Montanez, S. Moeller, D. Morton, H. D. Nuhn, N. Rodes, W. Schlotter, S. Serkez, T. Stevens, J. Turner, D. Walz, J. Welch, and J. Wu, Phys. Rev. Lett. 114, 054801 (2015).

14. D. Pile, Nat. Photonics 5, 456 (2011).

15. L. H. Yu, M. Babzien, I. Ben-Zvi, L. F. DiMauro, A. Doyuran, W. Graves, E. Johnson, S. Krinsky, R. Malone, I. Pogorelsky, J. Skaritka, G. Rakowsky, L. Solomon, X. J. Wang, M. Woodle, V. Yakimenko, S. G. Biedron, J. N. Galayda, E. Gluskin, J. Jagger, V. Sajaev, and I. Vasserman, Science 289, 932 (2000).
16. B. Liu, W. B. Li, J. H. Chen, Z. H. Chen, H. X. Deng, J. G. Ding, Y. Fan, G. P. Fang, C. Feng, L. Feng, Q. Gu, M. Gu, C. Guo, D. Z. Huang, M. M. Huang, W. H. Huang, Q. K. Jia, T. H. Lan, Y. B. Leng, D. G. Li, W. M. Li, X. Li, G. Q. Lin, L. Shang, L. Shen, C. X. Tang, G. L. Wang, L. Wang, R. Wang, X. T. Wang, Z. S. Wang, Z. S. Wang, H. F. Yao, K. R. Ye, L. X. Yin, L. Y. Yu, J. Q. Zhang, M. Zhang, M. Zhang, T. Zhang, W. Y. Zhang, S. P. Zhong, Q. G. Zhou, D. Wang, and Z. T. Zhao, Phys. Rev. ST Accel. Beams 16, 020704 (2013).

17. E. Allaria, P. Cinquegrana, S. Cleva, D. Cocco, M. Cornacchia, P. Craievich, I. Cudin, G. D’Auria, M. Dal Forno, M. Danailov, R. De Monte, G. De Ninno, P. Delgiusto, A. Demidovich, S. Di Mitri, B. Diviacco, A. Fabris, R. Fabris, W. Fawley, and M. Zangrando, Nat. Photonics 6, 699 (2012).

18. D. Xiang and G. Stupakov, Phys. Rev. ST Accel. Beams 12, 030702 (2009).

19. C. Feng, H. Deng, M. Zhang, X. Wang, S. Chen, T. Liu, K. Zhou, D. Gu, Z. Wang, Z. Jiang, X. Li, B. Wang, W. Zhang, T. Lan, L. Feng, B. Liu, Q. Gu, Y. Leng, L. Yin, D. Wang, Z. Zhao, G. Wang, and D. Xiang, Phys. Rev. Accel. Beams 22 , 050703 (2019).

20. Z. T. Zhao, D. Wang, J. H. Chen, Z. H. Chen, H. X. Deng, J. G. Ding, C. Feng, Q. Gu, M. M. Huang, T. H. Lan, Y. B. Leng, D. G. Li, G. Q. Lin, B. Liu, E. Prat, X. T. Wang, Z. S. Wang, K. R. Ye, L. Y. Yu, H. O. Zhang, J. Q. Zhang, M. Zhang, M. Zhang, T. Zhang, S. P. Zhong, and Q. G. Zhou, Nat. Photonics 6, 360 (2012).

21. P. R. Ribič, A. Abrami, L. Badano, M. Bossi, H.-H. Braun, N. Bruchon, F. Capotondi, D. Castronovo, M. Cautero, P. Cinquegrana, M. Coreno, M. E. Couprie, I. Cudin, M. B Danailov, G. De Ninno, A. Demidovich, S. Di Mitri, B. Diviacco, W. M. Fawley, C. Feng, M. Ferianis, E. Ferrari, L. Foglia, F. Frassetto, G. Gaio, D. Garzella, A. Ghaith, F. Giacuzzo, L. Giannessi, V. Grattoni, S. Grulja, E. Hemsing, F. Iazzourene, G. Kurdi, M. Lonza, N. Mahne, M. Malvestuto, M. Manfredda, C. Masciovecchio, P. Miotti, N. S. Mirian, I. P. Nikolov, G. M. Penco, G. Penn, L. Poletto, M. Pop, E. Prat, E. Principi, L. Raimondi, S. Reiche, E. Roussel, R. Sauro, C. Scafuri, P. Sigalotti, S. Spampinati, C. Spezzani, L. Sturari, M. Svandrlik, T. Tanikawa, M. Trovó, M. Veronese, D. Vivoda, D. Xiang, M. Zaccaria, D. Zangrando, M. Zangrando, and E. M. Allaria, Nat. Photonics 13, 555 (2019).

22. A. Wituschek, L. Bruder, E. Allaria, U. Bangert, M. Binz, R. Borghes, C. Callegari, G. Cerullo, P. Cinquegrana, L. Giannessi, M. Danailov, A. Demidovich, M. Di Fraia, M. Drabbels, R. Feifel, T. Laarmann, R. Michiels, N. S. Mirian, M. Mudrich, I. Nikolov, F. H. O'Shea, G. Penco, P. Piseri, O. Plekan, K. C. Prince, A. Przystawik, P. R. Ribič, G. Sansone, P. Sigalotti, S. Spampinati, C. Spezzani, R. J. Squibb, S. Stranges, D. Uhl, and F. Stienkemeier, Nat. Commun. 11, 883 (2020).

23. G. Penco, G. Perosa, E. Allaria, S. Di Mitri, E. Ferrari, L. Giannessi, S. Spampinati, C. Spezzani, and M. Veronese, Phys. Rev. Accel Beams 23, 120704 (2020).

24. Q. K. Jia, Nucl. Instrum. Methods Phys. Res. A Accel. Spectrom. Detect. Assoc. Equip. 1015, 165767 (2021).

25. S. Ackermann, B. Faatz, V. Grattoni, M. M. Kazemi, T. Lang, C. Lechner, G. Paraskaki, J. Zemella, G. Geloni, S. Serkez, T. Tanikawa, and W. Hillert, Phys. Rev. Accel. Beams 23, 071302 (2020).

26. G. Paraskaki, V. Grattoni, T. Lang, J. Zemella, B. Faatz, and W. Hillert, Phys. Rev. Accel. Beams 24, 034801 (2021).

27. D. J. Dunning, N. R. Thompson, and B. W. J. McNeil, J. Modern Opt. 58, 1362 (2011).

28. L. Zeng, W. L. Qin, G. Zhao, S. L. Huang, Y. T. Ding, Z. R. Huang, G. Marcus, and K. X. Liu, Chin. Phys. C 40, 098102 (2016). 
29. X. Wang, C. Feng, B. Faatz, W. Zhang, and Z. Zhao, arXiv:2103.11971 (2021).

30. M. Labat, M. Bellaveglia, M. Bougeard, B. Carré, F. Ciocci, E. Chiadroni, A. Cianchi, M. E. Couprie, L. Cultrera, M. Del Franco, G. Di Pirro, A. Drago, M. Ferrario, D. Filippetto, F. Frassetto, A. Gallo, D. Garzella, G. Gatti, L. Giannessi, G. Lambert, A. Mostacci, A. Petralia, V. Petrillo, L. Poletto, M. Quattromini, J. V. Rau, C. Ronsivalle, E. Sabia, M. Serluca, I. Spassovsky, V. Surrenti, C. Vaccarezza, and C. Vicario, Phys. Rev. Lett. 107, 224801 (2011).

31. J. Yan, Z. Gao, Z. Qi, K. Zhang, K. Zhou, T. Liu, S. Chen, C. Feng, C. Li, L. Feng, T. Lan, W. Zhang, X. Wang, X. Li, Z. Jiang, B. Wang, Z. Wang, D. Gu, M. Zhang, H. Deng, Q. Gu, Y. Leng, L. Yin, B. Liu, D. Wang, and Z. Zhao, Phys. Rev. Lett. 126, 084801 (2021).

32. J. W. Yan, N. S. Huang, H. X. Deng, B. Liu, D. Wang, and Z. T. Zhao, Adv. Photonics 3, 045003 (2021).

33. L. Giannessi, E. Allaria, L. Badano, D. Castronovo, P. Cinquegrana, P. Craievich, M. B. Danailov, G. D'Auria, A. Demidovitch, G. De Ninno, S. Di Mitri, B. Diviacco, W. M. Fawley, E. Ferrari, L. Froehlich, G. Gaio, R. Ivanov, E. Karantzoulis, B. Mahieu, N. Mahne, I. Nikolov, F. Parmigiani, G. Penco, L. Raimondi, C. Serpico, P. Sigalotti, S. Spampinati, C. Spezzani, M. Svandrlik, C. Svetina, M. Trovò, M. Veronese, D. Zangrando, M. Zangrando, in Proceedings of FEL2012 (2012), p. 13.

34. K.-J. Kim, Z. Huang, and R. Lindberg, Synchrotron Radiation and Free-Electron Lasers: Principles of Coherent X-Ray Generation (Cambridge University Press, Cambridge, 2017).
35. E. Prat, M. Calvi, R. Ganter, S. Reiche, T. Schietinger, and T. Schmidt, J. Synchrot. Radiat. 23, 861 (2016).

36. W. Qin, F. Curbis, J. Andersson, V. Goryashko, L. Isaksson, B. Kyle, F. Lindau, E. Mansten, M. Pop, P. Salen, H. Tarawneh, P. F. Tavares, S. Thorin, A. Vorozhtsov, and S. Werin, J. Synchrot. Radiat. 28, 707 (2021).

37. S. Reiche, Nucl. Instrum. Methods Phys. Res. Sect. A Accel. Spectrom. Detect. Associat. Equip. 429, 243 (1999).

38. R. Wilcox, G. Marcus, G. Penn, T. Metzger and M. Schultze, in Proceedings of FEL2013 (2013), p. 697.

39. V. Balandin and N. Golubeva, in Proceedings of 8 th International Particle Accelerator Conference (2017), p. 686.

40. P. Craievich, S. Di Mitri, M. Milloch, G. Penco, and F. Rossi, Phys. Rev. ST Accel. Beams 16, 090401 (2013).

41. P. Craievich, S. D. Mitri, and A. Zholents, in Proceedings of the 10th European Particle Accelerator Conference (2006), p. 2880 .

42. G. Penco, M. Danailov, A. Demidovich, E. Allaria, G. De Ninno, S. Di Mitri, W. M. Fawley, E. Ferrari, L. Giannessi, and M. Trovó, Phys. Rev. Lett. 112, 044801 (2014).

43. Z. Huang, D. Ratner, G. Stupakov. and D. Xiang, in Proceedings of FEL2009 (2009), p. 127.

44. K. Zhang, Z. Qi, C. Feng, H. Deng, D. Wang, and Z. Zhao, Nucl. Instrum. Methods Phys. Res. Sect. A Accel. Spectrom. Detect. Assoc. Equip. 854, 3 (2017).

45. N. Kroll, P. Morton, and M. Rosenbluth, IEEE J. Quantum. Elect. 17, 1436 (1981).

46. E. A. Schneidmiller and M. V. Yurkov, Phys. Rev. ST Accel. Beams 18, 030705 (2015). 